\title{
Placenta-associated serum exosomal miR-155 derived from patients with preeclampsia inhibits eNOS expression in human umbilical vein endothelial cells
}

\author{
LI SHEN $^{1 *}$, YUJING LI $^{1 *}$, RUOTIAN LI ${ }^{1}$, ZHENYU DIAO $^{1}$, MUYI YANY $^{1}$, \\ MENGFEI WU ${ }^{1}$, HAIXIANG SUN ${ }^{1}$, GUIJUN YAN ${ }^{1}$ and YALI HU ${ }^{1,2}$ \\ ${ }^{1}$ Reproductive Medicine Center, Drum Tower Clinic Medical College, Nanjing Medical University, Nanjing, \\ Jiangsu 210029; ${ }^{2}$ Molecular Reproductive Medical Center of Nanjing University, Nanjing, Jiangsu 210008, P.R. China
}

Received September 24, 2016; Accepted December 13, 2017

DOI: $10.3892 /$ ijmm.2018.3367

\begin{abstract}
Preeclampsia (PE) is considered to be initiated by abnormal placentation in early pregnancy and results in systemic endothelial cell dysfunction in the second or third trimester. MicroRNAs (miRs) expressed in the human placenta can be secreted into maternal circulation via exosomes, which are secreted extracellular vesicles that serve important roles in intercellular communication. The present study hypothesized that upregulation of placenta-associated serum exosomal miR-155 from patients with PE may suppress endothelial nitric oxide synthase (eNOS) expression in endothelial cells. The results demonstrated that placenta-associated serum exosomes from patients with PE decreased nitric oxide (NO) production and eNOS expression in primary human umbilical vein endothelial cells (HUVECs). Subsequently, an upregulation of placenta-associated serum exosomal miR-155 was detected in patients with PE compared with in gestational age-matched normal pregnant women. In addition, the results demonstrated that overexpression of exosomal miR-155 from BeWo cells was internalized into HUVECs, and was able to suppress eNOS expression by targeting its 3'-untranslated region. The results of the present study indicated that placenta-associated serum exosomes may inhibit eNOS expression in endothelial cell during PE development in humans, and this phenomenon may be partly due to increased miR-155 expression in placenta-associated serum exosomes.
\end{abstract}

Correspondence to: Professor Yali Hu, Reproductive Medicine Center, Drum Tower Clinical Medical College, Nanjing Medical University, 321 Zhongshan Road, Nanjing, Jiangsu 210029, P.R. China

E-mail: dtylhu@126.com

*Contributed equally

Key words: placenta-associated serum exosomes, microRNA-155, endothelial nitric oxide synthase, human umbilical vein endothelial cells, preeclampsia

\section{Introduction}

Preeclampsia (PE) is a pregnancy-specific complication associated with maternal and fetal morbidity and mortality; however, the exact etiology of this disease is not yet clearly understood (1). Until recently, PE has generally been described to be initiated by reduced placental perfusion, followed by impaired placentation, which in turn results in the release of molecules and factors leading to systemic endothelial activation and maternal clinical manifestations $(1,2)$.

Placental extracellular vesicles, including microvesicles, exosomes and syncytiotrophoblast-derived microparticles, can interact with endothelial cells and contribute to the development of PE $(3,4)$. Exosomes are nanovesicles $(30-100 \mathrm{~nm})$ that contain a wide range of functional proteins, mRNAs and microRNAs (miRNAs/miRs), and can be viewed as mediators of intercellular communication without the need for cell-cell contact $(5,6)$. There has been growing interest in exosomal miRNAs, since exosomal miRNA-mediated signaling has been reported to serve a significant role in various cell functions, including immune cell proliferation and endothelial cell migration $(7,8)$.

miRNAs are known to suppress gene expression post-transcription by binding the 3'-untranslated region (3'-UTR) of target mRNA. Numerous miRNAs have been demonstrated to affect placentation $(9,10)$, and miRNAs expressed in the placenta can be transferred into the maternal circulation via exosomes during human pregnancy (11). miR-155 is a well-known miRNA that possesses inflammatory and anti-angiogenic functions $(12,13)$. Our previous study, and other reported research, demonstrated that miR-155 is upregulated in the plasma and placentas from patients with PE, thus suggesting that miR-155 is associated with the pathogenesis of PE $(14,15)$.

Reduced nitric oxide (NO) bioavailability is involved in the pathophysiological alterations of $\mathrm{PE}$, and decreased endothelial nitric oxide synthase (eNOS) expression has been detected in the placentas and sera of patients with PE $(16,17)$. Our previous study indicated that miR-155 inhibits endothelium-dependent vasorelaxation by suppressing eNOS expression in human umbilical vein endothelial cells (HUVECs) (18). Therefore, it may be hypothesized that upregulation of placenta-associated 
serum exosomal miR-155 suppresses the expression of eNOS in endothelial cells during PE development.

The present study initially isolated and identified placenta-associated exosomes from the maternal sera of patients with PE and normal pregnant women. Subsequently, the effects of placenta-associated serum exosomes from patients with PE on NO production and eNOS expression in HUVECs were determined. In addition, the expression levels of miR-155 in these exosomes were detected. Finally, the expression of eNOS in endothelial cells treated with miR-155-overexpressed exosomes from BeWo cells was investigated.

\section{Materials and methods}

Human serum samples. Eligible participants were enrolled from the Nanjing Drum Tower Hospital (Nanjing, China) from May 1, 2015 to April 30, 2016. A total of 20 samples were obtained, 10 from patients with PE and 10 from gestational age-matched normal pregnant women (Table I). The diagnostic criteria of PE are described by Cunningham et al (19). Pregnant patients with PE alongside chronic hypertension, cardiovascular diseases, gestational diabetes mellitus or chronic nephritis were excluded from the present study. Sera samples $(2 \mathrm{ml})$ were obtained from each pregnant woman prior to treatment and were maintained at $-80^{\circ} \mathrm{C}$. The present study was approved by the Scientific Research Ethics Committee of the Drum Tower Hospital (2009041), and informed consent was obtained from all of the participants.

Cell lines. BeWo cells (GDC072) were purchased from the China Center for Type Culture Collection (CCTCC, Wuhan, China). Cells were maintained at $37^{\circ} \mathrm{C}$ in a humidified incubator containing $5 \% \mathrm{CO}_{2}$ and were cultured in RPMI-1640 medium (HyClone; GE Healthcare Life Sciences, Logan, UT, USA) supplemented with $10 \%$ heat-inactivated fetal bovine serum (FBS; HyClone; GE Healthcare Life Sciences), $100 \mathrm{U} / \mathrm{ml}$ penicillin and $100 \mu \mathrm{g} / \mathrm{ml}$ streptomycin. COS-7 cells (Thermo Fisher Scientific, Inc., Waltham, MA, USA) were cultured as described previously (18).

HUVEC isolation. Primary HUVECs were isolated from the vascular wall of umbilical veins obtained from normal pregnant women in the third trimester (not from the normal group). HUVECs were extracted by treatment of umbilical veins with $0.2 \%$ collagenase I (Worthington Biochemical Corporation,

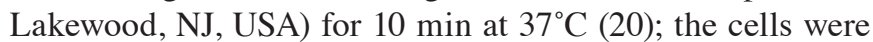
then seeded into $0.15 \%$ gelatin-coated plates (Sigma-Aldrich; Merck KGaA, Darmstadt, Germany) and were cultured for no more than six generations in M199 medium (HyClone; GE Healthcare Life Sciences) containing 20\% FBS and antibiotics (100 U/ml penicillin and $100 \mu \mathrm{g} / \mathrm{ml}$ streptomycin) at $37^{\circ} \mathrm{C}$ in a humidified incubator containing $5 \% \mathrm{CO}_{2}$.

Adenovirus and plasmid construction.pcDNA3-eNOS-3'-UTR plasmid and Ad-miR-155 adenovirus were constructed as previously described (18). Ad-LacZ served as the control for Ad-miR-155 infection. The COS-7 cells were plated and cultured overnight in the 6-well plates and at 70-80\% confluence, the cells were transfected with pcDNA-eNOS-3'-UTR $(2.0 \mu \mathrm{g})$ using Lipofectamine 2000 transfection reagent (Life
Table I. Clinical characteristics of patients with PE and normal pregnant women.

\begin{tabular}{lccc}
\hline Characteristic $^{\mathrm{a}}$ & $\begin{array}{c}\text { Normal } \\
(\mathrm{n}=10)\end{array}$ & $\begin{array}{c}\text { PE } \\
(\mathrm{n}=10)\end{array}$ & P-value \\
\hline Maternal age (years) & $27.56 \pm 3.21$ & $29.11 \pm 5.01$ & n.s. \\
Gestational age (weeks) & $39.14 \pm 0.66$ & $38.30 \pm 2.15$ & n.s. \\
${\text { Systolic BP }(\mathrm{mmHg})^{\mathrm{b}}}$ & $120.11 \pm 8.78$ & $149.56 \pm 17.17$ & $<0.05$ \\
${\text { Diastolic BP }(\mathrm{mmHg})^{\mathrm{b}}}$ & $80.22 \pm 8.50$ & $97.67 \pm 6.95$ & $<0.05$ \\
Proteinuria $^{\mathrm{b}}$ & - & +-+++ & $<0.05$ \\
${\text { AST }(\mathrm{U} / \mathrm{l})^{\mathrm{b}}}_{\text {ALT }(\mathrm{U} / \mathrm{l})^{\mathrm{b}}}$ & $10.28 \pm 1.87$ & $12.42 \pm 3.51$ & n.s. \\
Cr $(\mu$ mol/l) & $18.75 \pm 2.96$ & $24.13 \pm 7.60$ & n.s. \\
Platelets $\left(\mathrm{x} 10^{\mathrm{b}} / \mathrm{l}\right)^{\mathrm{b}}$ & $49.5 \pm 7.05$ & $42.6 \pm 1.34$ & $\mathrm{n} . \mathrm{s}$. \\
Fetal weight $(\mathrm{g})$ & $3,252.22 \pm 377.97$ & $2,822.78 \pm 400.67$ & $<0.05$ \\
Placenta weight $(\mathrm{g})$ & $630.00 \pm 76.03$ & $548.33 \pm 56.01$ & $<0.05$
\end{tabular}

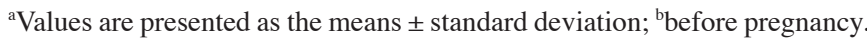
ALT, alanine aminotransferase; AST, aspartate aminotransferase; BP, blood pressure; Cr, creatinine; n.s., not significant; PE, preeclampsia.

Technologies, Grand Island, NY, USA). The relative intensity of eNOS protein expression was assessed by Image J $2 \mathrm{X}$ software (National Institutes of Health, Bethesda, MD, USA).

Exosome isolated from human peripheral sera. Exosomes were obtained by a series of centrifugation steps, combined with polyethylene glycol (PEG) precipitation. Briefly, serum $(200 \mu \mathrm{l})$ was centrifuged at $3,000 \mathrm{x}$ for $20 \mathrm{~min}$ at $4^{\circ} \mathrm{C}$, after which the supernatant was passed through a $0.22-\mu \mathrm{m}$ filter and was incubated with 8\% PEG 6000 overnight $(21,22)$. Following further centrifugation at $10,000 \mathrm{x}$ g for $1 \mathrm{~h}$ at $4^{\circ} \mathrm{C}$, the $8 \%$ PEG 6000-serum mixture precipitate was resuspended in $0.25 \mathrm{M}$ sucrose. Subsequently, the suspension was layered onto a linear sucrose gradient, ultracentrifuged at 100,000 x g for $5 \mathrm{~h}$ at $4^{\circ} \mathrm{C}$ and divided into six fractions: $\mathrm{F} 1$ (supernatant), 1.03; F2, 1.06; F3, 1.09; F4, 1.11; F5, 1.14; and F6, $1.18 \mathrm{~g} / \mathrm{ml}$. These fractions were incubated with 8\% PEG 6000 overnight and were then centrifuged at $10,000 \times \mathrm{g}$ for $1 \mathrm{~h}$ at $4^{\circ} \mathrm{C}$. Finally, the exosomes were suspended in $200 \mu \mathrm{l}$ PBS. An equal volume of sample from each fraction was used for western blotting. Sonicated exosomes were the control group since the sonicate would damage the exosomes.

Exosome isolation from culture medium. BeWo cells $\left(1.5 \times 10^{6}\right)$ were plated into a $100-\mathrm{mm}$ plate, infected with Ad-miR-155 or Ad-LacZ (100 multiplicity of infection) for $8 \mathrm{~h}$ at $37^{\circ} \mathrm{C}$ and were then incubated in medium containing $2 \%$ exosome-free FBS for a further $48 \mathrm{~h}$ at $37^{\circ} \mathrm{C}$. The culture supernatants were sequentially centrifuged at $300 \mathrm{x} \mathrm{g}$ for $10 \mathrm{~min}$ at $4^{\circ} \mathrm{C}$, $2,000 \mathrm{x} \mathrm{g}$ for $10 \mathrm{~min}$ at $4^{\circ} \mathrm{C}$ and $10,000 \mathrm{x} \mathrm{g}$ for $30 \mathrm{~min}$ at $4^{\circ} \mathrm{C}$. Subsequently, the cleared supernatants were ultracentrifuged at $100,000 \mathrm{x}$ g for $70 \mathrm{~min}$ at $4^{\circ} \mathrm{C}$ and the pellets were collected. Finally, the pellet suspension was ultracentrifuged for a second time at $100,000 \mathrm{x}$ g for $70 \mathrm{~min}$ at $4^{\circ} \mathrm{C}$ and the pellets were resuspended in $200 \mu \mathrm{l}$ PBS (23).

Primary HUVECs $\left(1.0 \times 10^{5}\right)$ in 12 -well plates were incubated with serum exosomes or with exosomes obtained from 
Table II. Oligonucleotide primer sequences for quantitative polymerase chain reaction.

\begin{tabular}{lll}
\hline Gene & \multicolumn{1}{c}{ Forward primer $\left(5^{\prime}-3^{\prime}\right)$} & Reverse primer $\left(5^{\prime}-3^{\prime}\right)$ \\
\hline miR-155 & CGTTAATGCTAATCGTGATAGG & GCAGGGTCCGAGGT \\
miR-39 & TCACCGGGTGTAAATCAG & GCAGGGTCCGAGGT \\
U6 & CTCGCTTCGGCAGCACA & AACGCTTCACGAATTTGCGT \\
eNOS & CCCTTCAGTGGCTGGTACAT & CACGATGGTGACTTTGGCTA \\
\hline
\end{tabular}

eNOS, endothelial nitric oxide synthase; miR, microRNA.

BeWo cell supernatants $(5.0 \mu \mathrm{g}$ protein $/ \mathrm{ml})$ in exosome-free medium for the indicated durations.

Transmissionelectronmicroscopy(TEM).Exosome-TEM-easy kit (cat. no. P130; Seajet Scientific, Inc., Beijing, China) was used to detect exosomes using Formvar/carbon-coated TEM grids (24). Freshly isolated exosome suspension (5-10 $\mu \mathrm{l}$ ) was dropped onto clean parafilm. Subsequently, the grids were floated on the drop with the coated side facing the suspension; the grid membranes were allowed to absorb exosomes for $10 \mathrm{~min}$. The grids were then transferred to the wash buffer for $30 \mathrm{sec}$, and were washed twice. The grids were then transferred to EM solution for $10 \mathrm{~min}$, and were washed twice. Finally, the grids were transferred to filter paper, coated side up, and were air dried overnight. The exosomes were visualized under TEM (JEM-1010; JEOL, Ltd., Tokyo, Japan) and 10 images were analyzed from each sample.

Dynamic light scattering (DLS). DLS measurements were performed using a Zetasizer 3000-HA (Malvern Instruments Ltd., Malvern, UK). Exosome samples were diluted in PBS and $0.05 \%$ Tween-20 to a total volume of $1.0 \mathrm{ml}$. The samples were diluted with PBS (1:10, 1:100 and 1:1,000). The samples with various dilutions in a total volume of $1.0 \mathrm{ml}$ were performed with standard settings (refractive index, 1.330; viscosity, 0.89; temperature, $25^{\circ} \mathrm{C}$ ) in the software.

Exosome labeling. Exosomes from BeWo cells or serum exosomes $(5.0 \mu \mathrm{g}$ protein $/ \mathrm{ml}$ used to treat HUVECs in 12-well plates) were resuspended in PBS (1 ml) and mixed with the green fluorescent dye PKH67 (Sigma-Aldrich; Merck KGaA) in $1 \mathrm{ml}$ Diluent $\mathrm{C}, 4 \times 10^{-6} \mathrm{M}(25)$. After a $3 \mathrm{~min}$ incubation, $1 \%$ bovine serum albumin $(2 \mathrm{ml})$ was added to terminate the labeling reaction, and the exosomes were harvested and washed twice with PBS by centrifugation $(100,000 \mathrm{x} \mathrm{g}$ for $1 \mathrm{~h})$. The exosomes were resuspended in medium ( $1 \mathrm{ml}$ ) supplemented with $10 \%$ ultrasonicated FBS and were then added to HUVECs in 12-well plates for $6 \mathrm{~h}$ incubation. The cells were washed three times with PBS, fixed with $4 \%$ paraformaldehyde for $30 \mathrm{~min}$ at room temperature, and were then stained with DAPI (1:5,000; Invitrogen; Thermo Fisher Scientific, Inc.) for $5 \mathrm{~min}$. After washing with PBS, the cells were visualized under a fluorescence microscope (Leica DMR; Leica Microsystems GmbH, Wetzlar, Germany).

Western blotting. Western blotting was performed according to standard procedures (26). Equal amounts of protein preparations were separated by SDS-PAGE, transferred to polyvinylidene fluoride membranes, immunoblotted with primary antibodies and visualized using a chemiluminescence detection kit (EMD Millipore, Bedford, MA, USA). The primary antibodies were as follows: Anti-eNOS (c-20; 1:1,000; sc-654) and anti-cluster of differentiation (CD)81 (1.3.3.22; 1:1,000; sc-7637) (both from Santa Cruz Biotechnology, Inc., Dallas, TX, USA), anti-placental alkaline phosphatase (PLAP; 1:1,000; AB21938b; Sangon Biotech Co., Ltd., Shanghai, China), anti-CD63 (K156; 1:1,000; BS3474) and anti- $\alpha$-tubulin (G436; 1:5,000; BS1699) (both from Bioworld Technology Inc., St. Louis Park, MN, USA). For the examination of CD63, CD81 and PLAP levels in the serum exosomes from patients with $\mathrm{PE}$, an equal volume of sample from each fraction was respectively used for western blotting, referring to the study by Putz et al (24). The expression levels of CD81, CD63 and PLAP were detected to be the highest level in the F3 fraction, indicating that placenta-associated serum exosomes were mostly accumulated in that fraction. For the examination of the levels of CD63, CD81 and PLAP in exosomes obtained from cell medium, an equal total protein of exosomes from the Ad-LacZ-infected cell medium and Ad-miR-155-infected cell medium were loaded for western blotting. The protein concentrations of the isolated exosomes from 2 groups were detected (Ad-LacZ-exosome, $0.35 \mu \mathrm{g} / \mu \mathrm{l}$; Ad-miR-155-exosome, $0.33 \mu \mathrm{g} / \mu \mathrm{l})$. And the total protein $(10 \mu \mathrm{g})$ from each group was loaded for western blotting to detect the expression levels of CD81, CD63 and PLAP.

Silver staining of polyacrylamide gels. The main steps of silver staining following PAGE (at room temperature) included: Fixing in acetic acid and ethanol mixture (v/v, 4/1) for $30 \mathrm{~min}$, permeabilization with $8 \mathrm{mM} \mathrm{Na}_{2} \mathrm{~S}_{2} \mathrm{O}_{3}$ for $30 \mathrm{~min}$ and staining with $7.5 \mathrm{mM} \mathrm{AgNO}_{3}$ in $37 \%$ formaldehyde solution for $30 \mathrm{~min}$. The bands would then be invisible when the gels were incubated in $0.25 \mathrm{M} \mathrm{Na}_{2} \mathrm{CO}_{3}$ for 2-15 min. Finally, $50 \mathrm{mM}$ glycine solution was added to terminate the staining process for $10 \mathrm{~min}$.

$R N A$ extraction and reverse transcription-quantitative polymerase chain reaction ( $R T-q P C R)$. Total RNA was extracted from cells using TRIzol (Invitrogen; Thermo Fisher Scientific, Inc.) and was reverse transcribed into cDNA with primers in a $20 \mu \mathrm{l}$ reaction using a PrimerScript RT regent kit (Takara, Otsu, Japan). Total RNA was extracted from exosomes using an Ambion mirVana PARIS kit (Ambion; Thermo Fisher Scientific, Inc.) according to the manufacturer's protocol. 
A

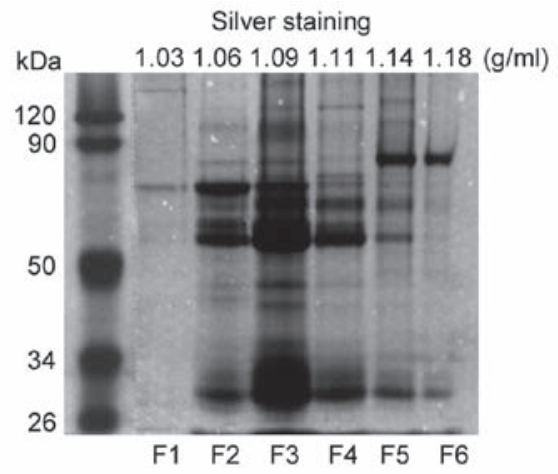

C

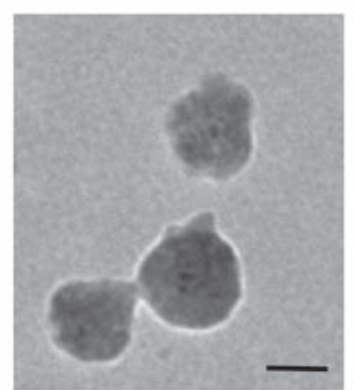

B

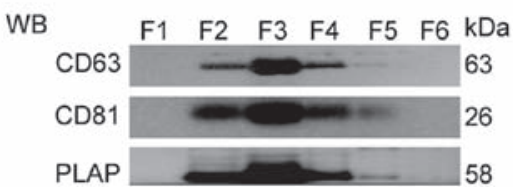

D

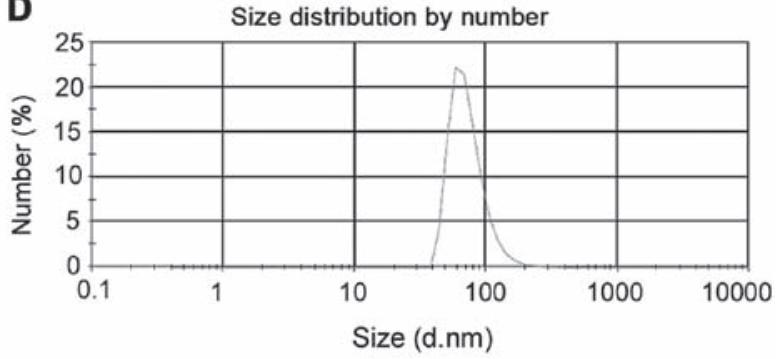

Figure 1. Characteristics of placenta-associated exosomes derived from the sera of patients with PE. (A) Isolated pelleted vesicles from sera of patients with PE were divided by sucrose gradients into six fractions, which were loaded onto gels for silver staining. (B) CD63, CD81 and PLAP were examined in the serum exosomes from patients with PE according to WB. (C) Electron microscopy images of purified exosomes in F3 derived from the sera of patients with PE. Scale bar, $50 \mathrm{~nm}$. (D) Particle size distributions of isolated exosomes in F3 were measured by dynamic light scattering. CD, cluster of differentiation; F, fraction; PE, preeclampsia; PLAP, placental alkaline phosphatase; WB, western blotting.

miR-39 (1.6x10 copy/ $\mu 1,2 \mu \mathrm{l}$; Qiagen, Hilden, Germany) was added to the exosomes prior to RNA isolation.

qPCR assays were performed as follows. Briefly, $20 \mathrm{ng}$ cDNA was applied to each $\mathrm{qPCR}$ reaction containing 2X SYBR-Green PCR Master Mix (Bio-Rad Laboratories, Inc., Hercules, CA, USA) and $500 \mathrm{nM}$ forward and reverse primers in a MyiQ Single-Color RT-PCR Detection system (Bio-Rad Laboratories, Inc.). RT-PCR for miR-155 was performed for 40 cycles $\left(95^{\circ} \mathrm{C}\right.$ for $10 \mathrm{sec} ; 60^{\circ} \mathrm{C}$ for $\left.30 \mathrm{sec}\right)$ following a initial 3 min incubation at $95^{\circ} \mathrm{C}$. RT-PCR for eNOS was performed via the blow procedure $\left(95^{\circ} \mathrm{C}\right.$ for $3 \mathrm{~min} ; 94^{\circ} \mathrm{C}$ for $10 \mathrm{sec} ; 60^{\circ} \mathrm{C}$ for $30 \mathrm{sec}, 72^{\circ} \mathrm{C}$ for $30 \mathrm{sec} ; 40$ cycles). The primers used are listed in Table II. The data obtained from three independent experiments were analyzed for relative gene expression using the $2^{-\Delta \Delta \mathrm{Cq}}$ method (27).

NO detection. NO levels were detected in the exosome-free culture medium of HUVECs treated with serum exosomes for $24 \mathrm{~h}$. NO concentration was measured using the NO detection kit (S0021; Beyotime Institute of Biotechnology, Shanghai, China) according to the manufacturer's protocol.

Statistical analysis. Normally distributed data were presented as the means \pm standard deviation using SPSS 17.0 software. The data obtained from three independent experiments were analyzed. Differences between means were analyzed using two-tailed Student's t-test, and one-way analysis of variance was used to detect differences among more than two groups. $\mathrm{P}<0.05$ was considered to indicate a statistically significant difference. The correlation between miR-155 expression in placenta-associated serum exosomal from patients with $\mathrm{PE}$ and
eNOS protein expression in HUVECs treated with placentaassociated serum exosomes was analyzed by GraphPad Prism 5.

\section{Results}

Characteristics of placenta-associated exosomes derived from the sera of patients with PE and normal pregnant women. Using sucrose gradients, isolated pelleted vesicles from the sera of patients with PE were separated into six fractions (F1-F6) of different densities. As shown in Fig. 1A, the pelleted vesicles in F3 $(1.09 \mathrm{~g} / \mathrm{ml})$ contained more bands than the other fractions, as determined by silver staining. Since tetraspanins are known to be expressed in exosomes (28), the present study examined CD63 and CD81 expression in the isolated serum fractions. As shown in Fig. 1B, CD63 and CD81 were detected in the pelleted vesicles in F2, F3 and F4; the expression of these molecules was highest in F3. Using PLAP as a marker for placental-derived exosomes (29), the present study confirmed that the vesicles in F3 contained the highest expression of PLAP. In addition, the present study measured the size of serum exosomes in F3 via TEM and DLS analysis. As presented in Fig. 1C, the diameter of isolated exosomes was $\sim 100 \mathrm{~nm}$. DLS analysis consistently demonstrated that the diameter of serum exosomes in F3 was $73.74 \pm 27.56 \mathrm{~nm}$ (Fig. 1D). The characteristics of placenta-associated serum exosomes from normal pregnant women are shown in Fig. 2.

Collectively, these data indicated that isolated vesicles in F3 derived from the peripheral sera of patients with PE and normal pregnant women contained a considerable amount of placenta-associated exosomes. The isolated vesicles in F3 were used for subsequent experiments. 
A
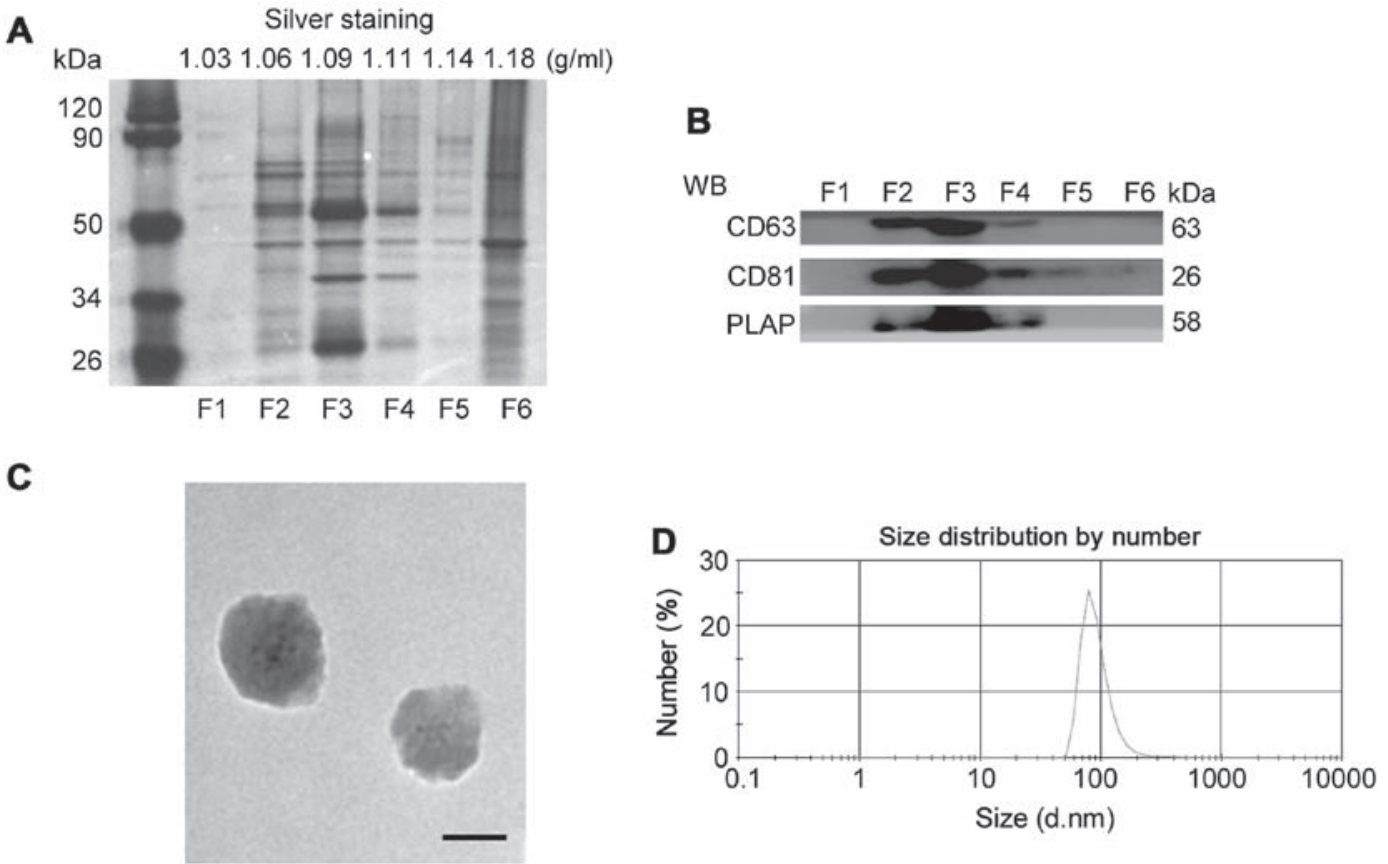

Figure 2. Characteristics of placenta-associated exosomes derived from sera of normal pregnant women in the third trimester. (A) Isolated pelleted vesicles were divided by sucrose gradients into six fractions, which were loaded onto gels for silver staining. (B) CD63, CD81 and PLAP were examined in the serum exosomes by WB. (C) Morphology of exosomes in F3 from normal pregnant women was observed by electron microscopy. Scale bar, 50 nm. (D) Particle size distributions of isolated exosomes in F3 were measured by dynamic light scattering. CD, cluster of differentiation; F, fraction; PE, preeclampsia; PLAP, placental alkaline phosphatase; WB, western blotting.

Placenta-associated serum exosomes from patients with PE decrease NO production and eNOS expression in HUVECs. The present study investigated the effects of serum exosomes on NO production and eNOS expression in HUVECs. Although the morphology of serum exosomes from normal pregnant women was similar to that of exosomes from patients with $\mathrm{PE}$, the NO concentration in HUVECs treated with placenta-associated serum exosomes from patients with $\mathrm{PE}$ was significantly decreased $(2.48 \pm 0.89 \mu \mathrm{M}$ vs. $3.53 \pm 0.88 \mu \mathrm{M}$, Fig. $3 \mathrm{~A} ; \mathrm{P}<0.05)$ compared with those from normal pregnant women. The mRNA expression levels of eNOS in HUVECs incubated with placenta-associated serum exosomes from patients with $\mathrm{PE}$ were reduced by $31.04 \%$ (Fig. $3 \mathrm{~B} ; \mathrm{P}<0.05$ ). In addition, the protein expression levels of eNOS were significantly downregulated in HUVECs incubated with placenta-associated serum exosomes from patients with PE (Fig. 3C and D; $\mathrm{P}<0.05$ ). These data indicated that placenta-associated serum exosomes from patients with PE may inhibit the expression of eNOS and NO production in endothelial cells.

miR-155 expression is increased in placenta-associated serum exosomes from patients with PE. Our previous study indicated that miR-155 is upregulated in the placentas of patients with PE (19). Therefore, the present study aimed to determine whether increased miR-155 expression was transferred via exosomes from the placenta into maternal circulation during pregnancy. Using RT-qPCR, it was demonstrated that miR-155 expression was increased in placenta-associated serum exosomes from patients with PE compared with in those from normal pregnant women (1.58-fold, P<0.05; Fig. 4A). Furthermore, an inverse correlation existed between miR-155 expression in placenta-associated serum exosomes from patients with PE and eNOS protein expression in HUVECs treated with placenta-associated serum exosomes $(\mathrm{r}=-0.5401$, $\mathrm{P}<0.05$; Fig. 4B).

miR-155-overexpressed exosomes from trophoblast cell medium inhibit eNOS expression in HUVECs in vitro. BeWo cells were infected with either Ad-miR-155 or Ad-LacZ, and exosomes were isolated from the culture medium. The characteristics of cell medium exosomes were identified, as follows: CD63, CD81 and PLAP were expressed in the isolated exosomes from BeWo cell medium, as determined by western blotting; and the diameter of the isolated exosomes was $\sim 100 \mathrm{~nm}$ (Fig. 5). Compared with exosomes from Ad-LacZ-infected cell medium, miR-155 expression was increased by 2.41-fold in exosomes from Ad-miR-155-infected cell medium (Fig. 6A; $\mathrm{P}<0.05$ ). The ability of exosomes to transfer to HUVECs was determined by examining the uptake of PKH67-labeled exosomes. Fluorescence microscopy demonstrated that PKH67-labeled BeWo cell exosomes were taken up by HUVECs and were distributed within the perinuclear compartments (Fig. 6B). As depicted in Fig. 6C, the expression levels of miR-155 were 4.93 -fold higher $(\mathrm{P}<0.05)$ in HUVECs treated with exosomes isolated from Ad-miR-155-infected BeWo cells compared with in those treated with Ad-LacZ-infected BeWo cell exosomes.

To examine whether exosomal miR-155 could suppress eNOS expression via direct 3'-UTR interaction, the present study investigated eNOS expression in pcDNA3-eNOS-3'-UTR-transfected COS-7 cells treated with exosomes from Ad-miR-155-infected BeWo cells (18). As shown in Fig. 6D, miR-155-overexpressed exosomes inhibited eNOS protein expression in a dose-dependent 


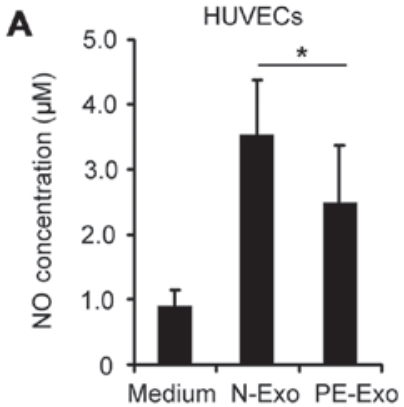

C

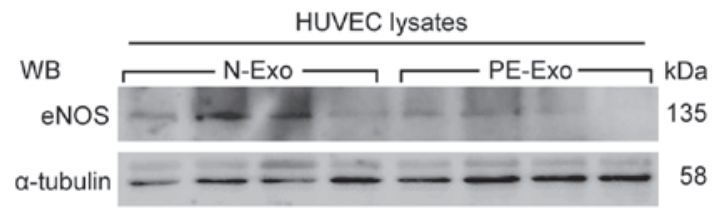

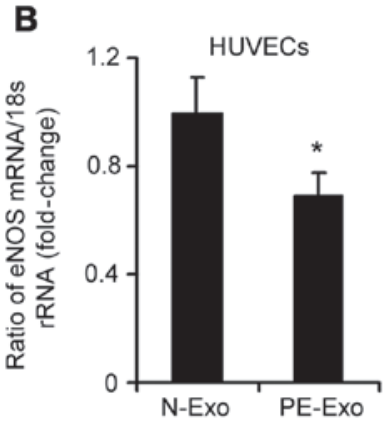

D

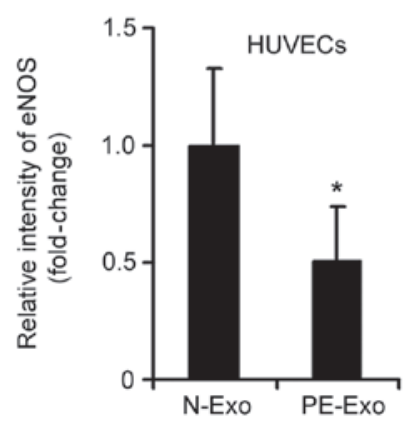

Figure 3. Placenta-associated serum exosomes from patients with PE inhibit NO production and eNOS expression in HUVECs. HUVECs were treated with serum exosomes $(5.0 \mu \mathrm{g} / \mathrm{ml})$ for $24 \mathrm{~h}$. Serum exosomes from gestational age-matched normal pregnant women served as a control. (A) NO levels were downregulated in the medium of HUVECs treated with placenta-associated serum exosomes from patients with PE. (B) eNOS mRNA and (C and D) protein expression levels were decreased in HUVECs treated with placenta-associated serum exosomes from patients with PE. The relative intensity of eNOS protein expression was assessed by ImageJ software. " $\mathrm{P}<0.05$ vs. N-Exo, $\mathrm{n}=10$ samples in the group. eNOS, endothelial NO synthase; HUVECs, human umbilical vein endothelial cells; N-Exo, exosomes from normal pregnant women; NO, nitric oxide; PE, preeclampsia; PE-Exo, exosomes from women with PE; WB, western blotting.
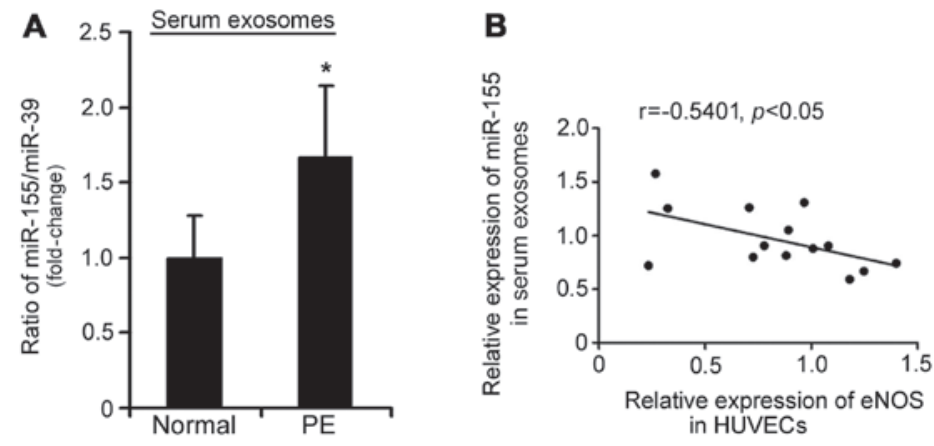

Figure 4. miR-155 is increased in placenta-associated serum exosomes from patients with PE. (A) miR-155 was upregulated in placenta-associated serum exosomes from patients with PE. Quantitative polymerase chain reaction was used to detect miR-155 expression. $\mathrm{n}=10$ samples in the group. ${ }^{*} \mathrm{P}<0.05 \mathrm{vs}$. normal group. (B) Correlation between the expression of miR-155 in placenta-associated serum exosomes from patients with PE and eNOS protein expression in HUVECs treated with exosomes, as analyzed by GraphPad Prism 5. eNOS, endothelial nitric oxide synthase; HUVECs, human umbilical vein endothelial cells; miR, microRNA; PE, preeclampsia;
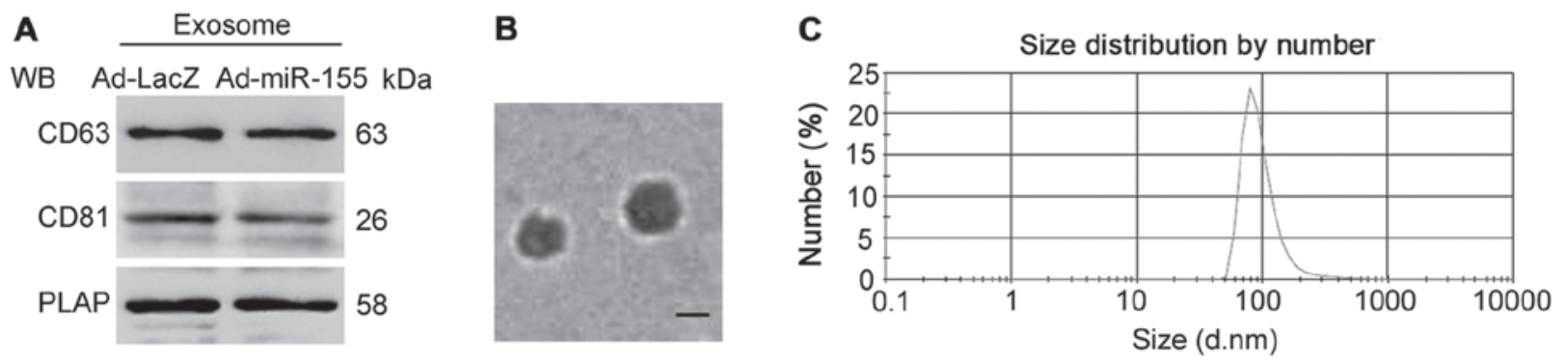

Figure 5. Characteristics of exosomes derived from Ad-infected BeWo cell medium. (A) CD63, CD81 and PLAP were expressed in exosomes obtained from cell medium, as determined by western blotting. (B) Electron microscopy image of purified exosomes derived from cell medium. Scale bar, $50 \mathrm{~nm}$. (C) Particle size distributions of isolated cell medium exosomes were measured by dynamic light scattering. (B and C) Data from exosomes derived from Ad-miR-155-infected cell medium. The morphology of serum exosomes from Ad-miR-155-infected cell medium was similar to that of exosomes from Ad-LacZ-infected cell medium. Ad, adenovirus; CD, cluster of differentiation; miR-155, microRNA-155; PLAP, placental alkaline phosphatase; WB, western blotting. 
A

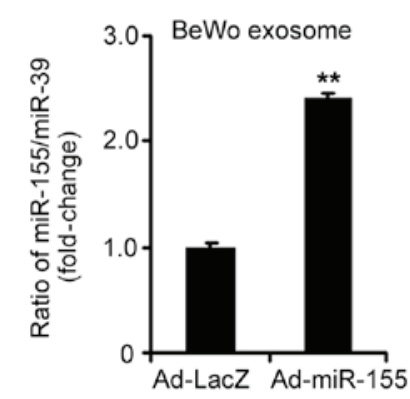

C
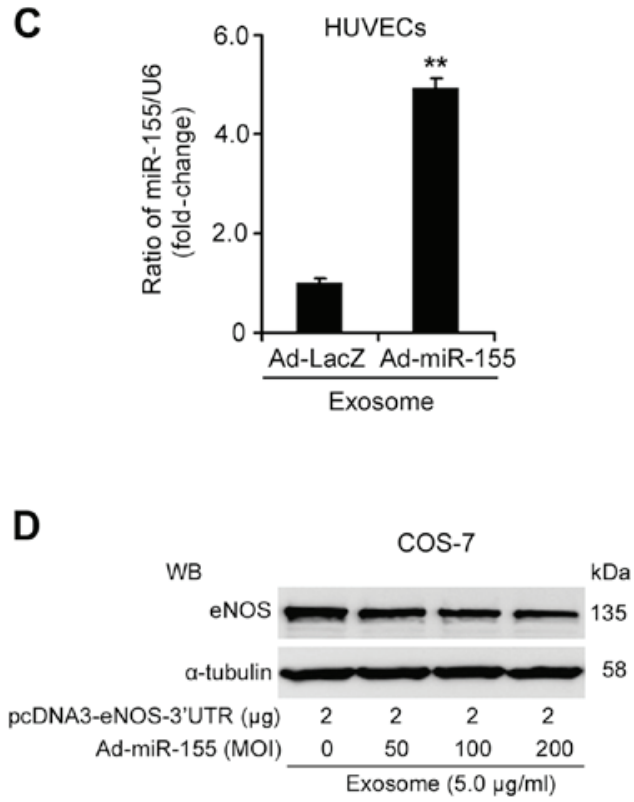

$\mathbf{F}$

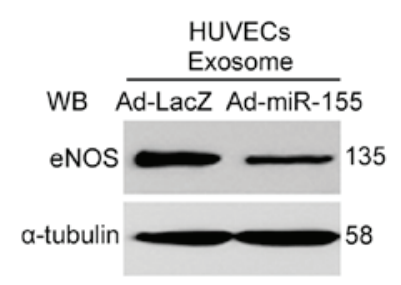

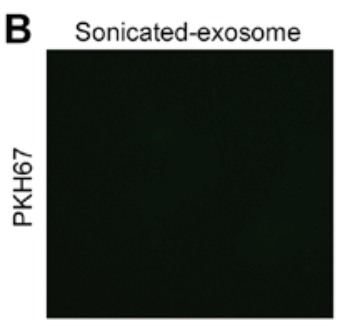
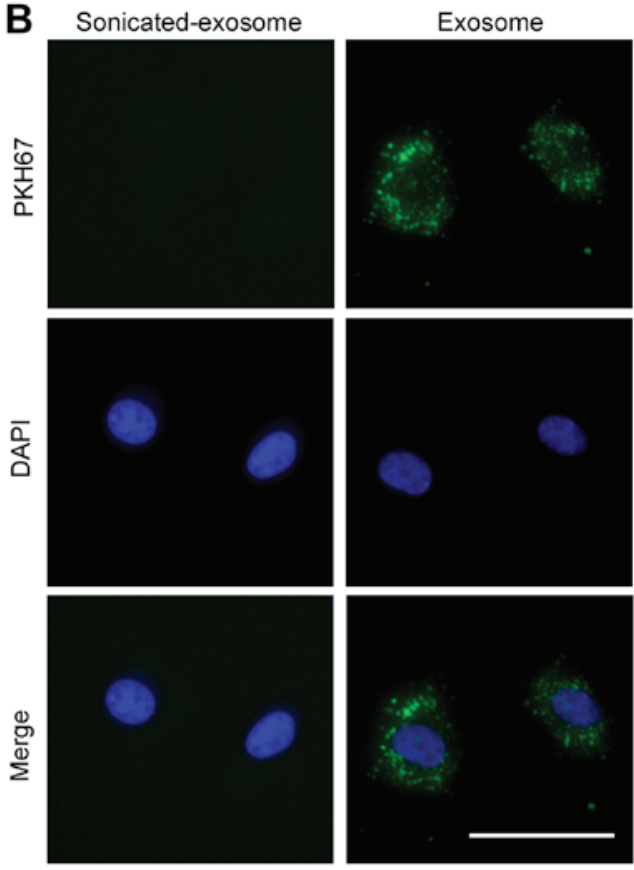

E

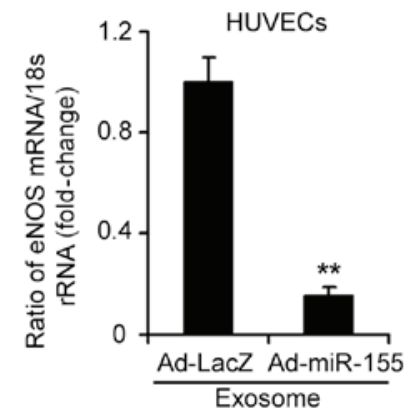

G

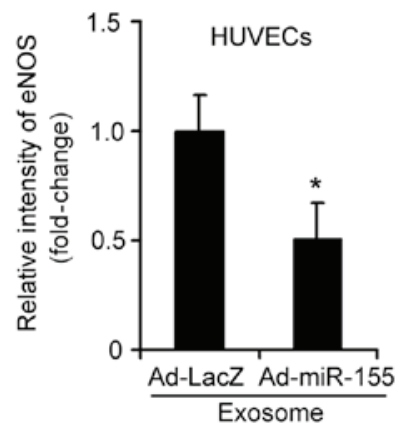

Figure 6. miR-155-overexpressed exosomes from BeWo cell medium suppress eNOS expression in HUVECs. (A) RT-qPCR analysis of miR-155 in exosomes from BeWo cells infected with Ad-miR-155 or Ad-LacZ. (B) HUVECs were incubated for $6 \mathrm{~h}$ with PKH67 fluorescently-labeled exosomes and were observed under a fluorescence microscope (PKH67, green; DAPI, blue). Scale bar, $25 \mu \mathrm{m}$. (C) Expression of miR-155 was increased in HUVECs treated with exosomes from Ad-miR-155-infected BeWo cells. HUVECs were treated with exosomes $(5.0 \mu \mathrm{g} / \mathrm{ml})$ for $24 \mathrm{~h}$. (D) miR-155-overexpressed exosomes from BeWo cells inhibited eNOS expression by targeting its 3'-UTR in COS-7 cells. The pcDNA3-eNOS plasmid containing the 3'-UTR of the eNOS gene was transfected into COS-7 cells for $24 \mathrm{~h}$, then miR-155-overexpressed exosomes from BeWo cells $(5.0 \mu \mathrm{g} / \mathrm{ml})$ were added to the transfected cells for $24 \mathrm{~h}$. (E) eNOS mRNA and $(\mathrm{F}$ and $\mathrm{G})$ protein levels were decreased in HUVECs treated with exosomes from Ad-miR-155-infected BeWo cells. eNOS expression was detected by RT-qPCR and WB. The relative intensity of eNOS protein expression was assessed using ImageJ software. These data were from three independent

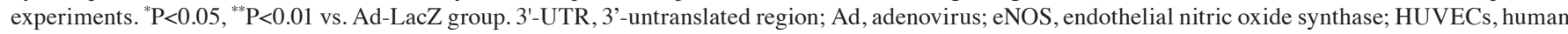
umbilical vein endothelial cells; miR-155, microRNA-155; RT-qPCR, reverse transcription-quantitative polymerase chain reaction; WB, western blotting.

manner. Subsequently, the present study investigated eNOS expression in HUVECs treated with exosomes from Ad-miR-155-infected BeWo cells. The mRNA expression levels of eNOS were reduced by $\sim 84.73 \%$ in HUVECs treated with exosomes from Ad-miR-155-infected BeWo cells (Fig. 6E; $\mathrm{P}<0.05$ ). Consistently, the protein expression levels of eNOS were markedly decreased in HUVECs incubated with miR-155-overexpressed exosomes compared with 
in those treated with exosomes from Ad-LacZ-infected BeWo cells (Fig. 6F and G; $\mathrm{P}<0.05$ ). Taken together, these results indicated that exosomal miR-155 derived from trophoblasts may suppress eNOS expression in HUVECs.

\section{Discussion}

During human pregnancy, placenta-associated serum exosomes are involved in cell-cell communication between the placenta and peripheral vascular endothelial cells (29). The present study isolated serum exosomes with PLAP-positive expression by PEG precipitation and ultracentrifugation with a sucrose gradient, which led to the isolation of placenta-associated exosomes with high purity and yield. The present study demonstrated that placenta-associated serum exosomes from patients with PE reduced NO production and eNOS expression in endothelial cells; this effect may be partly due to the increased expression of miR-155 in placenta-associated serum exosomes.

Previous studies have accumulated evidence regarding the physiological function of serum exosomal miRNAs. Squadrito et al demonstrated that serum/plasma exosomal miR-122 and miR-155 are predominantly associated with inflammatory liver injury (30). Another study indicated that serum exosomal miR-21 is positively correlated with tumor progression and aggressiveness (31). In addition, miRNAs in plasma exosomes have been reported to be stable under various storage conditions (32). These data have led to the recognition that serum exosomal miRNAs serve important roles in pathophysiological alterations and the pathogenesis of diseases. Placenta exosomal miRNAs may mediate crosstalk between the fetoplacental unit and the maternal system during pregnancy $(33,34)$. The present study initially detected the upregulation of miR-155 in placenta-associated serum exosomes from patients with PE compared with in those from normal pregnant women.

Endothelial cell functions can be affected by exosomes via various mechanisms $(9,35)$. Since vasomotor dysfunction is one of the pathological alterations associated with PE, the present study focused on vasomotor function, and demonstrated that placenta-associated serum exosomes from patients with $\mathrm{PE}$ exhibited downregulated eNOS expression and NO production in HUVECs. The expression of eNOS can be modulated at numerous levels, and post-transcriptional regulation has an important role in the modulation. The present study revealed the inhibitory effect of exosomal miR-155 on eNOS expression using pcDNA3-eNOS-3'-UTR-overexpressing COS-7 cells, which do not possess endogenous eNOS expression. In addition, miR-155-overexpressed exosomes isolated from trophoblast cell medium were able to suppress the expression of eNOS in HUVECs. Therefore, placenta-associated serum exosomal miR-155 may suppress eNOS expression through direct 3'-UTR interaction. These findings provided evidence to suggest that serum placenta-associated exosomes may regulate endothelial cell functions during PE development.

In conclusion, the present study identified that placenta-associated serum exosomes from patients with $\mathrm{PE}$ suppressed NO production and eNOS expression in endothelial cells. In addition, increased miR-155 expression in placenta-associated serum exosomes may be associated with the effects of serum exosomes. Our findings indicated that serum exosomal miR-155 may regulate endothelial cell functions and contribute to the development of PE.

\section{Acknowledgements}

The authors would like to thank Dr Zhiqun Wang and Dr Yimin Dai (both from Nanjing Drum Tower Hospital) for collection of human serum samples. The study was funded in part by grants from the National Natural Science Foundation (grant nos. 81370724, 81571462 and 81701472), the Jiangsu Biobank of Clinical Resources (grant no. BM2015004) and the Jiangsu Key Laboratory for Molecular Medicine (grant no. BM2007208), Project of Nanjing Clinical Medicine.

\section{References}

1. Redman CW and Sargent IL: Latest advances in understanding preeclampsia. Science 308: 1592-1594, 2005.

2. James JL, Whitley GS and Cartwright JE: Preeclampsia: fitting together the placental, immune and cardiovascular pieces. J Pathol 221: 363-378, 2010.

3. Redman CW, Tannetta DS, Dragovic RA, Gardiner C, Southcombe JH, Collett GP and Sargent IL: Review: does size matter? Placental debris and the pathophysiology of preeclampsia. Placenta 33 (Suppl): S48-S54, 2012.

4. Redman CW and Sargent IL: Placental debris, oxidative stress and preeclampsia. Placenta 21: 597-602, 2000.

5. Keller S, Sanderson MP, Stoeck A and Altevogt P: Exosomes: from biogenesis and secretion to biological function. Immunol Lett 107: 102-108, 2006.

6. Valadi H, Ekström K, Bossios A, Sjöstrand M, Lee JJ and Lötvall JO: Exosome-mediated transfer of mRNAs and microRNAs is a novel mechanism of genetic exchange between cells. Nat Cell Biol 9: 654-659, 2007.

7. Nazimek K, Ptak W, Nowak B, Ptak M, Askenase PW and Bryniarski K: Macrophages play an essential role in antigen-specific immune suppression mediated by $\mathrm{T}$ CD $8^{+}$ cell-derived exosomes. Immunology 146: 23-32, 2015.

8. Lee HD, Kim YH and Kim DS: Exosomes derived from human macrophages suppress endothelial cell migration by controlling integrin trafficking. Eur J Immunol 44: 1156-1169, 2014.

9. Chen DB and Wang W: Human placental microRNAs and preeclampsia. Biol Reprod 88: 130, 2013.

10. Hromadnikova I, Kotlabova K, Hympanova L and Krofta L: Cardiovascular and cerebrovascular disease associated microRNAs are dysregulated in placental tissues affected with gestational hypertension, preeclampsia and intrauterine growth restriction. PLoS One 10: $\mathrm{e} 0138383,2015$.

11. Luo SS, Ishibashi O, Ishikawa G, Ishikawa T, Katayama A, Mishima T, Takizawa T, Shigihara T, Goto T, Izumi A, et al: Human villous trophoblasts express and secrete placenta-specific microRNAs into maternal circulation via exosomes. Biol Reprod 81: 717-729, 2009.

12. Pankratz F, Bemtgen X, Zeiser R, Leonhardt F, Kreuzaler S, Hilgendorf I, Smolka C, Helbing T, Hoefer I, Esser JS, et al: MicroRNA-155 exerts cell-specific antiangiogenic but proarteriogenic effects during adaptive neovascularization. Circulation 131: 1575-1589, 2015.

13. Bala S, Csak T, Saha B, Zatsiorsky J, Kodys K, Catalano D, Satishchandran A and Szabo G: The pro-inflammatory effects of miR-155 promote liver fibrosis and alcohol-induced steatohepatitis. J Hepatol 64: 1378-1387, 2016.

14. Murphy MS, Casselman RC, Tayade C and Smith GN: Differential expression of plasma microRNA in preeclamptic patients at delivery and 1 year postpartum. Am J Obstet Gynecol 213: 367.e1-367.e9, 2015.

15. Zhang Y, Diao Z, Su L, Sun H, Li R, Cui H and Hu Y: MicroRNA-155 contributes to preeclampsia by down-regulating CYR61. Am J Obstet Gynecol 202: 466.e1-466.e7, 2010.

16. Johal T, Lees CC, Everett TR and Wilkinson IB: The nitric oxide pathway and possible therapeutic options in preeclampsia. Br J Clin Pharmacol 78: 244-257, 2014.

17. Kim YJ, Park HS, Lee HY, Ha EH, Suh SH, Oh SK and Yoo HS: Reduced L-arginine level and decreased placental eNOS activity in preeclampsia. Placenta 27: 438-444, 2006. 
18. Sun HX, Zeng DY, Li RT, Pang RP, Yang H, Hu YL, Zhang Q, Jiang Y, Huang LY, Tang YB, et al: Essential role of microRNA-155 in regulating endothelium-dependent vasorelaxation by targeting endothelial nitric oxide synthase. Hypertension 60: 1407-1414, 2012.

19. Cunningham FG, Leveno KJ, Bloom SL, Spong CY, Dashe JS, Hoffman BL, CaseyBM and Sheffield JS: Hypertensive disorders. In: Williams Obstetrics. 24th edition. McGraw-Hill Education, New York, NY, pp729-731, 2014.

20. Baudin B, Bruneel A, Bosselut $\mathrm{N}$ and Vaubourdolle M: A protocol for isolation and culture of human umbilical vein endothelial cells. Nat Protoc 2: 481-485, 2007.

21. Vlassov A, Li M, Zeringer E and Conrad R: Methods and compositions for exosome isolation. US Patent 8,901,284 B2. Filed February 26, 2014; issued December 2, 2014.

22. Rider MA, Hurwitz SN and Meckes DG Jr: ExtraPEG: a polyethylene glycol-based method for enrichment of extracellular vesicles. Sci Rep 6: 23978, 2016.

23. Li J, Liu K, Liu Y, Xu Y, Zhang F, Yang H, Liu J, Pan T, Chen J, Wu M, et al: Exosomes mediate the cell-to-cell transmission of IFN- $\alpha$-induced antiviral activity. Nat Immunol 14: 793-803, 2013

24. Putz U, Howitt J, Doan A, Goh CP, Low LH, Silke J and Tan SS The tumor suppressor PTEN is exported in exosomes and has phosphatase activity in recipient cells. Sci Signal 5: ra70, 2012.

25. Pegtel DM, Cosmopoulos K, Thorley-Lawson DA, van Eijndhoven MA, Hopmans ES, Lindenberg JL, de Gruijl TD, Würdinger T and Middeldorp JM: Functional delivery of viral miRNAs via exosomes. Proc Natl Acad Sci USA 107: 6328-6333, 2010.

26. Hnasko TS and Hnasko RM: The western bot. Methods Mol Biol 318: 87-96, 2015

27. Livak KJ and Schmittgen TD: Analysis of relative gene expression data using real-time quantitative PCR and the 2(-Delta Delta C(T)) Method. Methods 25: 402-408, 2001.
28. Rana S, Yue S, Stadel D and Zöller M: Toward tailored exosomes: the exosomal tetraspanin web contributes to target cell selection. Int J Biochem Cell Biol 44: 1574-1584, 2012.

29. Salomon C, Torres MJ, Kobayashi M, Scholz-Romero K Sobrevia L, Dobierzewska A, Illanes SE, Mitchell MD and Rice GE: A gestational profile of placental exosomes in maternal plasma and their effects on endothelial cell migration. PLoS One 9: e98667, 2014

30. Squadrito ML, Baer C, Burdet F, Maderna C, Gilfillan GD, Lyle R, Ibberson $M$ and De Palma M: Endogenous RNAs modulate microRNA sorting to exosomes and transfer to acceptor cells. Cell Reports 8: 1432-1446, 2014.

31. Tanaka Y, Kamohara H, Kinoshita K, Kurashige J, Ishimoto T, Iwatsuki $\mathrm{M}$, Watanabe $\mathrm{M}$ and Baba $\mathrm{H}$ : Clinical impact of serum exosomal microRNA-21 as a clinical biomarker in human esophageal squamous cell carcinoma. Cancer 119: $1159-1167,2013$.

32. Ge Q, Zhou Y, Lu J, Bai Y, Xie X and Lu Z: miRNA in plasma exosome is stable under different storage conditions. Molecules 19: 1568-1575, 2014.

33. Ouyang Y, Mouillet JF, Coyne CB and Sadovsky Y: Review: placenta-specific microRNAs in exosomes - good things come in nano-packages. Placenta 35 (Suppl): S69-S73, 2014.

34. Kambe S, Yoshitake H, Yuge K, Ishida Y, Ali MM, Takizawa T, Kuwata T, Ohkuchi A, Matsubara S, Suzuki M, et al: Human exosomal placenta-associated miR-517a-3p modulates the expression of PRKG1 mRNA in Jurkat cells. Biol Reprod 91: $129,2014$.

35. Gambim MH, do Carmo AO, Marti L, Veríssimo-Filho S, Lopes LR and Janiszewski M: Platelet-derived exosomes induce endothelial cell apoptosis through peroxynitrite generation: experimental evidence for a novel mechanism of septic vascular dysfunction. Crit Care 11: R107, 2007. 